\title{
北疆地区15种豆科植物种子对绵羊消化道作用的 响应
}

\section{王树林 鲁为华 ${ }^{*}$ 陈乙实 景鹏成}

石河子大学动物科技学院, 新疆石河子 832003

摘 要 为揭示北疆地区豆科植物种子对绵羊消化道作用的响应, 采集了北疆地区天然草地中常见的 15 种豆科植物种子。首 先测定种子的长、宽、厚和质量, 并计算种子的形状指数。其次, 用种子饲喂绵羊, 收粪试验在种子摄食后的第6、12、24、 36、 48 和72 h 进行, 测定种子经绵羊消化道作用后的回收率、种子在绵羊消化道内的平均滞留时间以及消化道作用前后种子 的萌发行为, 并研究了种子大小及形状指数对平均滞留时间和种子回收率的影响。结果如下: 15 种植物种子质量在1.50-37.68 $\mathrm{mg}$ 之间, 形状指数在0.001-0.12之间, 均为中等或较大类型的球(圆)形种子; 种子被绵羊采食后的排放动态符合高斯模型: $Y=0.02+0.74 \mathrm{e}^{-0.5((X-29.61) / 9.41)^{2}}$, 种子排放高峰期集中在采食后的 $24-36 \mathrm{~h}$ 时间段内; 种子回收率最大的是洋甘草(Glycyrrhiza glabra)(39.25\%), 最小的是草木犀(Melilotus officinalis)(4.28\%); 平均滞留时间最长的是草木犀 $(37.19 \mathrm{~h})$, 最短的是新疆棘豆 (Oxytropis sinkiangensis)(22.33 h); 种子回收率与种子大小和形状指数之间分别具有符合形如 $Y=6.45+2.05 X-0.04 X^{2}$ 和 $Y=$ $2.59+36.97 \mathrm{e}^{-24.47 X}$ 的函数关系模型; 平均滞留时间与种子大小和形状指数之间分别具有符合形如 $Y=12.48+37.44 \mathrm{e}^{-0.07 X}$ 和 $Y=$ $3.93+2055.33 X-21757.99 X^{2}$ 的函数关系模型, 此结果表明, 较大、较小和形状不规则的豆科种子较易被绵羊消化和排泄。经 绵羊消化道作用后, 多叶锦鸡儿(Caragana pleiophylla)种子的萌发率由消化前的 $96.22 \%$ 显著降低至 $35.17 \%$, 野火球(Trifolium lupinaster)和狐尾黄耆(Astragalus alopecurus)种子萌发率和消化前相比差异不显著, 其余12种种子的萌发率均显著提高 $(p<$ 0.05)。

关键词 种子消化道传播; 北疆地区; 豆科植物; 种子质量; 形状指数; 滞留时间; 种子回收率

王树林, 鲁为华, 陈乙实, 景鹏成 (2018). 北疆地区 15 种豆科植物种子对绵羊消化道作用的响应. 植物生态学报, 42, 185-194. DOI: $10.17521 /$ cjpe. 2017.0118

\section{Effect of sheep digestive tract on the recovery and germination of seeds of fifteen leguminous plants in the northern Xinjiang region, China}

WANG Shu-Lin, LU Wei-Hua ${ }^{*}$, CHEN Yi-Shi, and JING Peng-Cheng

College of Animal Science and Technology, Shihezi University, Shihezi, Xinjiang 832003, China

\begin{abstract}
Aims The objective of this study was to learn the effect of sheep digestive tract on the recovery and germination of seeds of fifteen leguminous plants in the northern Xinjiang region.

Methods The seeds were collected from fifteen leguminous species which were widely distributed in the natural mowing pasture of the northern Xinjiang region. After determining their morphological characteristics (seed length, width, thickness, single seed mass, and seed shape index), seeds were fed to sheep. Faeces collection was carried out at $6,12,24,36,48$ and $72 \mathrm{~h}$ after seeds were eaten by sheep. Seed recovery percentage (SRP), mean retention time (MRT) and seed germinability before and after ingested were determined, and the relationships between seed size, seed shape index and SRP, MRT after ingested were also studied.

Important findings Seed mass ranged from 1.50 to $37.68 \mathrm{mg}$, and seed shape index between 0.001 and 0.12 , which indicated the seeds are all medium or large type sphericity (round) seeds. Seed excretion dynamic followed a Gaussian model: $Y=0.02+0.74 \mathrm{e}^{-0.5((X-29.61) / 9.41)^{2}}\left(R^{2}=0.62, p<0.01\right)$, and excretion peak appeared $24-36 \mathrm{~h}$ after digestion. Seed recovery rate was ranged from $39.25 \%$ (Glycyrrhiza glabra) to $4.28 \%$ (Melilotus officinalis). The MRT ranged from $37.19 \mathrm{~h}$ (Melilotus officinalis) to $22.33 \mathrm{~h}$ (Oxytropis sinkiangensis). The relationship
\end{abstract}

收稿日期Received: 2017-05-16 接受日期Accepted: 2018-01-04

基金项目: 国家自然科学基金(31560659和31360568)。Supported by the National Natural Science Foundation of China (31560659 and 31360568 ).

* 通信作者Corresponding author (winnerlwh@sina.com; 2294997914@qq.com) 
between $S R P$ and seed size is expressed as the equation $Y=6.45+2.05 X-0.04 X^{2}\left(R^{2}=0.41, p<0.05\right)$. The relationship between seed $S R P$ and seed shape index is expressed as the equation $Y=2.59+36.97 \mathrm{e}^{-24.47 X}\left(R^{2}=0.37, p\right.$ $<0.05)$. The relationship between $M R T$ and seed size is expressed as the equation $Y=12.48+37.44 \mathrm{e}^{-0.07 X}\left(R^{2}=\right.$ $0.37, p<0.05)$. The relationship between $M R T$ and seed shape index is expressed as the equation $Y=3.93+$ $2055.33 X-21757.99 X^{2}\left(R^{2}=0.42, p<0.05\right)$. The results suggested that leguminous seeds in larger, or smaller, or irregular shape were more easily digested and excreted by sheep. After digested by sheep gut, germination percentages of recovered seeds of Caragana pleiophylla (35.17\%) was significantly decreased $(p<0.05)$ compared with that of seeds before ingested (96.22\%). Similarly, seed germination percentages of Trifolium lupinaster and Astragalus alopecurus seeds were decreased than those of no ingested seeds (not significantly, $p>0.05$ ), but the germination percentages of other twelve kinds seeds were significantly increased $(p<0.05)$.

Key words seed endozoochore dispersal; northern Xinjiang region; leguminous plant; seed mass; shape index; retention time; seed recovery percentage

Wang SL, Lu WH, Chen YS, Jing PC (2018). Effect of sheep digestive tract on the recovery and germination of seeds of fifteen leguminous plants in the northern Xinjiang region, China. Chinese Journal of Plant Ecology, 42, 185-194. DOI: 10.17521/cjpe.2017.0118

豆科植物因其具有适口性好、蛋白含量高、营 养丰富、消化率高、各类家畜喜欢采食的特点而被 称为饲用植物中的“肉类” (赵丽丽等, 2007)。新疆天 然草地中的豆科植物有 400 余种, 多数分布于北疆 地区, 它们中的大部分种类是优等牧草, 是北疆地 区放牧家畜重要的饲草来源(于否等, 2014)。豆科植 物各物种之间由于分布地域不同, 在生物学特性和 饲用价值上存在明显的多样性特征，尤其是种子形 态学特征和传播方式上差异更大, 因此野生豆科植 物种子生物学特性, 尤其在种子形态学特征、传播 方式及萌发行为等方面一直是种子生物学研究的热 点内容。

种子消化道传播(seed endozoochore dispersal)是 指植物种子被食草(果)动物采食, 经过消化道作用 以后, 通过反刍或粪便排出体外从而实现传播的现 象(Oudtshoorn \& Rooyen, 1998), 动物对植物种子 的消化道传播是种子实现远距离传播的重要方式。 种子由于动物采食、经消化道携带和排泄而产生空 间上的大尺度位移, 一方面为植物种群开拓新栖息 地创造了有利条件、避免了同胞间激烈的竞争, 另 一方面大大增加了空间尺度上的景观多样性 (Heidrun \& Oliver, 2008)。目前关于草食动物对植物 种子消化道传播的研究, 所涉及的动物种类有牛 (Willms et al., 1995)、绵羊(Ramos et al., 2006)、山羊 (Mancilla-Leytón et al., 2012)、马(Milotić \& Hoffmann, 2016)和驴(Couvreur et al., 2005)等放牧家畜。 研究的生态类型包括森林(Jaroszewicz, 2013)、草原 (Myers et al., 2004)和沼泽(Brochet et al., 2010)等。 具体研究内容包括: 种子自身形态学特征与种子被
消化道作用后的回收率、活力以及在消化道内的滞 留时间之间的关系(Blackshaw \& Rode, 1991), 排出 后的种子萌发所形成的幼苗的生长状况以及消化道 传播对植被更新过程的影响(Plumptre, 2003), 并且 基于上述研究内容可进一步探讨植物与草食动物二 者之间是否存在协同进化(D’Hondt \& Hoffmann, 2011)。

Janzen (1984)通过对草食动物对植物种子的消 化道传播进行研究提出了著名的“双F假说(foliage is the fruit hypothesis)”。该假说认为植物的叶片和茎 秆等营养器官作为草食动物的食物来源, 二者在动 植物之间的关系上与肉质果所具有的营养丰富的果 肉对动物的采食引诱是等同的, 这种策略是植物为 了使种子被动物采食实现传播而进行的间接的繁殖 投资(Janzen, 1984)。天然草地中豆科植物多为草本 (陈本建, 2008), 且种子与植物的叶片、茎秆等可食 部位在空间位置上联系紧密, 这为豆科植物种子被 家畜采食并通过消化道传播创造了便利条件。此外, 豆科植物种子普遍存在休眠现象(娜丽克斯 - 外里等, 2016), 而动物在采食植物种子时伴随咀嚼和体内 消化液消化种子等过程。因此种子在动物体内的滞 留能够打破某些具有休眠特性的种子的休眠, 提高 其萌发率(Gökbulak, 2006)。这也为草食动物对豆科 植物种子成功地进行消化道传播提供了可能。目前 国内关于草食动物对植物种子的消化道传播的研究 所涉及的物种数量较少, 仅包括禾本科(杨洁晶等, 2015)和十字花科中某些能分泌黏液的种子(王树林 等, 2017)等少数科属的植物种子。研究内容只局限 于动物消化道的某一特定部位(如瘤胃(娜丽克斯 - 外

www.plant-ecology.com 
里等, 2016))对种子命运的影响, 并没有包括动物消 化道作用的全过程。北疆地区作为我国重要的放牧 畜牧业经营地, 开展此区域的植物种子消化道传播 的研究对于北疆地区家畜的放牧管理和退化植被的 自然更新与恢复具有重要意义。

在野外实地观察中发现, 各类家畜(牛、马和羊) 均喜好采食豆科植物植株, 其粪便中也能发现完整 的种子, 且粪便分布范围内聚集有豆科植物的幼苗 或成株。以上现象说明豆科植物种子被家畜采食和 排泄后仍具有活力。由此推测, 家畜的取食偏好和 消化行为对豆科种子传播和萌发过程具有一定的影 响。为了验证北疆地区豆科植物种子对放牧绵羊消 化道作用的响应，以广布于北疆地区各大草场的 15 种豆科植物种子为研究对象, 通过种子形态测量和 动物(绵羊)饲喂试验, 旨在明确以下问题: 1)这 15 种 豆科植物的种子大小和形状指数如何? 2)种子大小 及形状指数与种子在绵羊体内的平均滞留时间以及 种子经绵羊消化后的回收率之间具有怎样的关系?

$3)$ 这 15 种豆科植物是否可以通过放牧绵羊进行种子 的消化道传播?

\section{1 材料和方法}

\section{1 试验材料}

供试种子采集于天山北坡中段山地 $\left(84.97^{\circ}-\right.$ $86.40^{\circ} \mathrm{E}, 43.43^{\circ}-45.33^{\circ} \mathrm{N}$, 海拔 $1935-2252 \mathrm{~m}$ )、塔 城地区的巴尔鲁克山天然割草场 $\left(82.35^{\circ}-82.41^{\circ} \mathrm{E}\right.$, $45.57^{\circ}-45.58^{\circ} \mathrm{N}$, 海拔1 100-1600 m) 和伊犁沙尔套 山的山前倾斜平原(山麓带) $\left(80.15^{\circ}-80.54^{\circ} \mathrm{E}, 42.54^{\circ}-\right.$ $43.11^{\circ} \mathrm{N}$, 海拔2 200-2 $300 \mathrm{~m}$ ), 共计 15 种, 分别是 洋甘草(Glycyrrhiza glabra)、草木犀(Melilotus officinalis)、苦马豆(Sphaerophysa salsula)、蒙古黄耆 (Astragalus mongholicus)、木黄耆(Astragalus arbuscula)、新疆棘豆(Oxytropis sinkiangensis)、多叶 锦鸡儿(Caragana pleiophylla)、野火球 (Trifolium lupinaster)、骆驼刺(Alhagi sparsifolia)、山羊豆 (Galega officinalis)、铃铛刺 (Halimodendron halodendron)、野苜宿(Medicago falcata)、细叶野踠豆 (Vicia tenuifolia)、狐尾黄耆(Astragalus alopecurus) 和弯果胡卢巴(Trigonella arcuata)。按适口性等级划 分, 这 15 种豆科植物均属于优级或良级牧草, 即适 口性极好, 一般情况下各类家畜均喜食或从草地中 优先挑选采吃(于否等, 2014)。根据不同植物种子的
成熟时期，于2015年6-10月间进行种子采集。种子 采集后放在室内通风处自然风干后，装入信封袋内 于 $4{ }^{\circ} \mathrm{C}$ 冰箱中保存备用。

\section{2 试验方法}

\subsection{1 种子形态学指标的观测}

将每种植物种子混合均匀, 从中挑选籽粒饱满 的种子 10 粒, 分别用 $1 / 10000$ 电子天平称其质量, 重复 5 次, 共称取 50 粒种子的单粒质量 $(S M)$, 然后取 平均值作为每种种子的单粒质量。将种子大小(种子 质量)分成5个等级, 即 $\mathrm{A}$ 级: $S M<0.1 \mathrm{mg}$; B级: $0.1 \mathrm{mg}$ $\leqslant S M<1.0 \mathrm{mg}$; C级: $1.0 \mathrm{mg} \leqslant S M<10 \mathrm{mg}$; D级: $10 \mathrm{mg} \leqslant S M<100 \mathrm{mg}$; E级: $S M \geqslant 100 \mathrm{mg}$, 作为 种子大小的量化依据。每种植物再挑选 10 粒正常大 小的种子, 用电子游标卡尺和实体显微镜(Nikon SMZ1500, 上海千欣仪器有限公司)测量种子的长 度、宽度和高(厚)度，参考Thompson等(1993)的计算 方法, 计算每种种子的形状指数, 即将种子形状与 球体对比, 求出种子长、宽和高的总体方差(公式 (1))。方差越小, 种子越接近圆球形; 方差越大, 种 子越细长或扁平。在计算方差前对数据进行转换, 方法是先将长定为 1 , 然后求出宽和高相对于长的 比值。

$$
I=\frac{\left[3\left(X_{l}{ }^{2}+X_{w}{ }^{2}+X_{h}{ }^{2}\right)-\left(X_{l}+X_{w}+X_{h}\right)^{2}\right]}{3^{2}}
$$

式中 $I$ 为种子形状指数, $X_{1} 、 X_{\mathrm{w}}$ 和 $X_{\mathrm{h}}$ 为种子的长、宽 和高三维指标经过转换后的数值。

\subsection{2 种子饲喂及粪便回收}

选用3只体重为 $(25.00 \pm 2.68) \mathrm{kg}$ 的哈萨克公羊, 分别在具有粪尿分离装置的代谢笼内单笼饲养。饲 喂种子前预饲一周, 每只羊日粮组成为 $200 \mathrm{~g}$ 精料 + $1.8 \mathrm{~kg}$ 苜宿干草(除净种子), 自由饮水。之后开始种 子饲喂试验(饲喂试验从2016年11月5日开始，为期 100 天)。每只羊一次饲喂一种种子(2000粒)。为便 于种子被绵羊采食, 饲喂时将种子与精料混合均匀, 实验中观察到大约 $40 \mathrm{~min}$ 后种子被全部取食。从种 子被羊全部取食完开始，分别在之后的6、12、24、 36、48和 $72 \mathrm{~h}$ 用阶段性全收粪法(Manzano et al., 2005; 娜丽克斯 - 外里等, 2016)收集每只羊排出的 粪便。一种种子饲喂及粪便收集完成 5 天后再进行下 一次种子饲喂和粪便收集工作，直至所有种子的饲 喂及粪便收集工作完成为止。收集的粪样做好标记, 带回实验室自然晾干。然后对不同时间段的每一只 
羊的所有粪样称质量, 混合均匀后从中取出 $600 \mathrm{~g}$ 作 为次级样品, 手工将其碾碎, 利用水淘法分离出其 中的所有种子。种子回收试验完成后, 分别用公式 (2)和(3)计算经绵羊消化道作用后的种子回收率 $(S R P)$ 和种子在绵羊体内的平均滞留时间 $(M R T)$, 其 公式 如下:

$$
\begin{aligned}
& \operatorname{SRP}(\%)=\left(\frac{M f \times S r}{600 \times S}\right) \times 100 \% \\
& M R T=\sum_{i=1}^{n} m_{i} t_{i} / \sum_{i=1}^{n} m_{i}
\end{aligned}
$$

式中, $M f$ 为不同时间段的总粪样, $S r$ 为每 $600 \mathrm{~g}$ 粪样 中所包含的种子数, $S$ 为被羊取食的总的种子数量; $m_{i}$ 指绵羊在摄入种子的 $t_{i}$ 时刻第 $i$ 次排便时所释放的 种子数, $n$ 为排便次数。

\subsection{3 种子萌发试验}

用从绵羊粪便中回收到的各种种子为实验组进 行萌发实验(100粒), 以原始种子为对照(CK), 采用 纸上法(韩建国，2007)，2017年2月20号开始种子萌 发实验。将种子放入铺有双层滤纸的培养血中, 每 个培养血中放 100 粒。将放有种子的培养血置于 GXZ型智能光照培养箱中进行萌发试验。根据采集 地植被生长的气候条件设置培养箱的温变周期为 $8 \mathrm{~h}$ $15{ }^{\circ} \mathrm{C} / 16 \mathrm{~h} 25{ }^{\circ} \mathrm{C}$ 变温和光照, 每 $24 \mathrm{~h}$ 进行萌发检测, 统计萌发个数, 胚根出现 $2 \mathrm{~mm}$ 即可认为已经萌发。 萌发实验共进行 30 天，按照公式(4)计算种子萌发率:

萌发率 $(\%)=$ (萌发种子数 $/$ 种子总数 $) \times 100 \%(4)$

\section{3 数据处理}

所有数据均采用Excel 2007整理, 用SigmaPlot 12.5软件作图, 用DPS 7.5软件进行数据的单因素方 差分析, 多重比较采用最小显著差数法 $(L S D)$, 实验 结果均以平均值土标准误差表示。

\section{2 结果和分析}

\subsection{5 种豆科植物种子的单粒质量和形状指数}

对种子质量进行统计, 结果表明 15 种豆科植物 种子的平均质量为 $10.73 \mathrm{mg}$, 但种间差异较大, 单 粒质量最大的是多叶锦鸡儿(37.68 mg), 最小的是 弯果胡卢巴 $(1.50 \mathrm{mg})$, 二者相差 25 倍。单粒质量属 于D级(10 mg $\leqslant S M<100 \mathrm{mg}$ )的有5种, 分别是蒙 古黄耆、多叶锦鸡儿、铃铛刺、细叶野踠豆和狐尾黄 耆; 其余 10 种种子的单粒质量均属于C级 $(1.0 \mathrm{mg} \leqslant$ $S M<10 \mathrm{mg}$ )(表1)。这15种种子均为中等或较大类型 的种子。

根据种子的长、宽和高对种子的形状指数进行 计算, 结果表明 15 种豆科植物种子形状指数的平均 值为 0.07 , 种子形状指数最大的是木黄耆 $(0.12)$, 最 小的是洋甘草和细叶野踠豆, 均为 0.001 , 除蒙古黄 耆、木黄耆和弯果胡卢巴 3 种种子外, 其余 12 种种子 的形状指数均 $<0.1$, 占物种总数的 $80 \%$ (表 1 )。

\subsection{5 种豆科植物种子饲喂绵羊后的排放动态}

对 15 种豆科植物种子经绵羊采食后种子的回收 数量随收粪时间的变化进行非线性拟合，拟合前对 数据进行标准化处理以消除不同种子间回收数量的 量级差异，具体方法是用每个时间段的种子回收数 量除以所有种子回收数量中的最大值, 使所有种子 回收数量值均在 0-1范围内 (娜丽克斯 - 外里等, 2016)。结果表明这 15 种豆科种子的排放动态符合高 斯模型: $Y=0.02+0.74 \mathrm{e}^{-0.5((X-29.61) / 9.41)^{2}}\left(R^{2}=\right.$ $0.62)($ 图1), 决定系数达到极显著水平 $(p<0.05)$ 。绵 羊对种子的排放动态为单峰曲线, 随时间的延长种 子回收数量呈现先增高后降低的趋势。种子被采食 后的前 $6 \mathrm{~h}$ 内几乎没有种子排出, 至 $12 \mathrm{~h}$ 已有少量种

表1 15 种豆科种子的单粒质量及种子形状指数(平均值土标准误差)

\begin{tabular}{|c|c|c|c|c|c|}
\hline $\begin{array}{l}\text { 种名 } \\
\text { Species }\end{array}$ & $\begin{array}{c}\text { 单粒质量 } \\
\text { Single seed mass (mg) }\end{array}$ & $\begin{array}{l}\text { 形状指数 } \\
\text { Shape index }\end{array}$ & $\begin{array}{c}\text { 种名 } \\
\text { Species }\end{array}$ & $\begin{array}{c}\text { 单粒质量 } \\
\text { Single seed mass (mg) }\end{array}$ & $\begin{array}{c}\text { 形状指数 } \\
\text { Shape index }\end{array}$ \\
\hline 洋甘草 Glycyrrhiza glabra & $6.69 \pm 0.03$ & $0.001 \pm 0.000$ & 骆驼刺 Alhagi sparsifolia & $4.47 \pm 0.03$ & $0.070 \pm 0.007$ \\
\hline 草木犀 Melilotus officinalis & $2.17 \pm 0.02$ & $0.050 \pm 0.010$ & 山羊豆 Galega officinalis & $6.22 \pm 0.03$ & $0.070 \pm 0.006$ \\
\hline 苦马豆 Sphaerophysa salsula & $7.28 \pm 0.02$ & $0.070 \pm 0.010$ & 铃铛刺 Halimodendron halodendron & $15.73 \pm 0.05$ & $0.070 \pm 0.005$ \\
\hline 蒙古黄耆 Astragalus mongholicus & $10.59 \pm 0.02$ & $0.100 \pm 0.003$ & 野苜宿 Medicago falcata & $1.98 \pm 0.01$ & $0.070 \pm 0.010$ \\
\hline 木黄耆 Astragalus arbuscula & $3.72 \pm 0.03$ & $0.120 \pm 0.004$ & 细叶野豌豆 Vicia tenuifolia & $14.84 \pm 0.06$ & $0.001 \pm 0.001$ \\
\hline 新疆棘豆 Oxytropis sinkiangensis & $2.63 \pm 0.03$ & $0.070 \pm 0.001$ & 狐尾黄耆 Astragalus alopecurus & $34.42 \pm 0.12$ & $0.070 \pm 0.001$ \\
\hline 多叶锦鸡儿 Caragana pleiophylla & $37.68 \pm 0.07$ & $0.070 \pm 0.010$ & 弯果胡卢巴 Trigonella arcuata & $1.50 \pm 0.03$ & $0.100 \pm 0.008$ \\
\hline 野火球 Trifolium lupinaster & $1.67 \pm 0.01$ & $0.070 \pm 0.010$ & & & \\
\hline
\end{tabular}

Table 1 Single seed mass and seed shape index of seeds of fifteen leguminous species (mean $\pm S E$ ) 


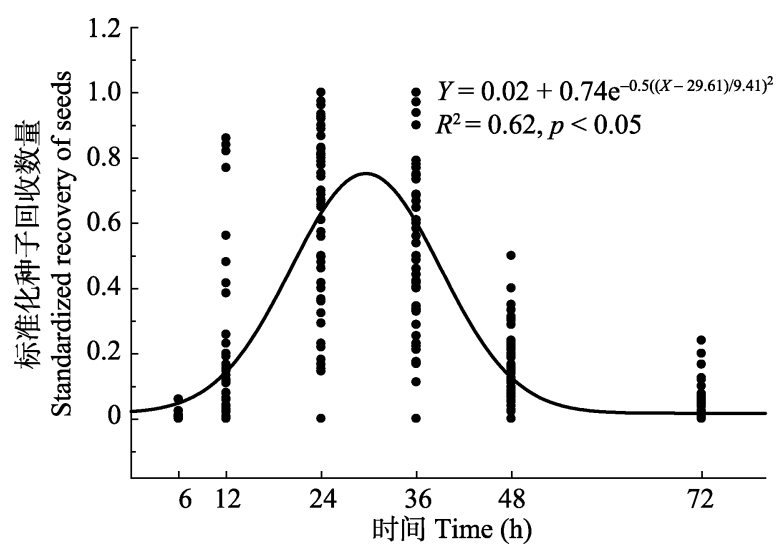

图1 种子饲喂给绵羊后的排放动态。

Fig. 1 Temporal changes in seed recovery after ingestion by sheep.

子排出, 在采食24-36 h 后种子排放达到高峰期, 超 过 $80 \%$ 的种子在此期间被排出, 至 $72 \mathrm{~h}$ 以后粪便内 几乎没有回收到种子。

\subsection{5 种豆科植物种子经绵羊消化后的SRP及种} 子在绵羊体内的 $M R T$

通过对种子经绵羊消化后的 $S R P$ 进行计算, 结 果表明, $S R P$ 最大的是洋甘草(39.25\%), 最小的是草 木犀(4.28\%), 二者相差9倍。SRP > 30\%的物种有 5 种, 分别是洋甘草、苦马豆、山羊豆、铃铛刺和细 叶野踠豆; $S R P$ 在 $10 \%-30 \%$ 之间的物种有 4 种, 分别 是新疆棘豆、野火球、骆驼刺和狐尾黄老; SRP < $10 \%$ 的物种有 6 种, 分别是草木犀、蒙古黄耆、木黄 耆、多叶锦鸡儿、野苜宿和弯果胡卢巴。单因素方 差分析结果显示, 不同种类的种子 $S R P$ 之间的差异 达到显著水平 $(p<0.05)$ (表 2$)$ 。

对 15 种豆科植物种子在绵羊消化道内的 $M R T$ 进 行计算, 结果表明 $M R T$ 最长的是草木犀 $(37.19 \mathrm{~h})$,
最短的是新疆棘豆 $(22.33 \mathrm{~h})$ 。 $M R T<30 \mathrm{~h}$ 的有 5 种, 分别是新疆棘豆、骆驼刺、山羊豆、野苜㑑和弯果 胡卢巴; 其余 10 种种子 $M R T$ 均 $>30 \mathrm{~h}$ 。单因素方差分 析结果显示, 不同种类的种子在绵羊体内的 $M R T$ 之 间的差异达到显著水平 $(p<0.05)$ (表2)。

\section{4 种子大小和形状指数与 $S R P$ 和 $M R T$ 的关系}

$S R P$ 随种子大小的递增呈现先增高后降低的趋 势; 随种子形状指数的增大逐渐减少, 即种子形状 越不规则, 被绵羊采食后的 $S R P$ 越低, 且 $S R P$ 与种子 大小和形状指数之间分别具有形如 $Y=6.45+2.05 X-$ $0.04 X^{2}\left(R^{2}=0.41\right)$ 和 $Y=2.59+36.97 \mathrm{e}^{-24.47 X}\left(R^{2}=\right.$ $0.37)$ 的函数关系模型, 相关系数均达到显著水平 $(p<$ 0.05 ), 表明拟合结果可信(图2)。

种子在绵羊体内的 $M R T$ 随种子大小的增加呈现 先增高后降低的趋势; 随种子形状指数的增加逐渐 减小, 表明越不规则的种子在绵羊体内的 $M R T$ 越长, 且 $M R T$ 与种子大小和形状指数之间分别具有形如 $Y=$ $26.14+0.70 X-0.01 X^{2}\left(R^{2}=0.37\right)$ 和 $Y=34.71-$ $179.78 X+736.10 X^{2}\left(R^{2}=0.42\right)$ 的函数关系模型, 相 关系数均达到显著水平 $(p<0.05)$, 表明拟合结果可 信(图2)。

\section{5 绵羊消化道作用前后种子的萌发率}

将种子萌发率进行分组, 包括高萌型(萌发率> $80 \%)$, 中间型 $(55 \%-80 \%)$, 次低萌型 $(20 \%-55 \%)$, 低萌型(萌发率 $<20 \%$ )。绵羊消化道作用前, 属于高 萌型的种子有 1 种, 为多叶锦鸡儿(96.22\%); 属于次 低型的种子有 3 种, 分别是木黄耆 $(45.00 \%)$ 、新疆棘 豆 $(33.33 \%)$ 和野火球 $(28.33 \%)$; 其余 11 种种子的萌 发率均 $<20 \%$, 属于低萌型种子(表3)。

种子经绵羊消化后, 萌发率最高的是苦马豆

表2 15 种豆科种子经绵羊采食后的种子回收率及种子在绵羊体内的平均滞留时间(平均值沶准误差)

Table 2 Seed recovery percentages and mean retention time of 15 leguminous species after sheep ingestion (mean $\pm S E)$

\begin{tabular}{|c|c|c|c|c|c|}
\hline $\begin{array}{l}\text { 种名 } \\
\text { Species }\end{array}$ & $\begin{array}{c}\text { 种子回收率 } \\
\operatorname{SRP}(\%)\end{array}$ & $\begin{array}{c}\text { 平均滞留时间 } \\
M R T(\mathrm{~h})\end{array}$ & $\begin{array}{l}\text { 种名 } \\
\text { Species }\end{array}$ & $\begin{array}{c}\text { 种子回收率 } \\
\operatorname{SRP}(\%)\end{array}$ & $\begin{array}{c}\text { 平均滞留时间 } \\
M R T(\mathrm{~h})\end{array}$ \\
\hline 洋甘草 Glycyrrhiza glabra & $39.25 \pm 20.40^{\mathrm{a}}$ & $31.62 \pm 0.73^{\mathrm{bcd}}$ & 骆驼刺 Alhagi sparsifolia & $25.06 \pm 9.25^{\mathrm{ab}}$ & $28.27 \pm 0.27^{\mathrm{bcd}}$ \\
\hline 草木犀 Melilotus officinalis & $4.28 \pm 1.76^{\mathrm{c}}$ & $37.19 \pm 2.12^{\mathrm{ab}}$ & 山羊豆 Galega officinalis & $38.02 \pm 9.60^{\mathrm{a}}$ & $27.55 \pm 1.75^{\text {bcd }}$ \\
\hline 苦马豆 Sphaerophysa salsula & $38.60 \pm 19.77^{\mathrm{a}}$ & $32.48 \pm 0.39^{\mathrm{abcd}}$ & 铃铛刺 Halimodendron halodendron & $34.56 \pm 15.89^{\mathrm{a}}$ & $36.51 \pm 5.93^{\mathrm{ab}}$ \\
\hline 蒙古黄耆 Astragalus mongholicus & $4.88 \pm 1.02^{\mathrm{c}}$ & $36.62 \pm 0.54^{\mathrm{ab}}$ & 野苜蕧 Medicago falcata & $9.41 \pm 1.26^{\mathrm{bc}}$ & $24.58 \pm 0.84^{\mathrm{cd}}$ \\
\hline 木黄耆 Astragalus arbuscula & $5.02 \pm 1.25^{\mathrm{c}}$ & $32.51 \pm 3.93^{\mathrm{abcd}}$ & 细叶野踠豆 Vicia tenuifolia & $34.90 \pm 2.27^{\mathrm{a}}$ & $32.78 \pm 2.94^{\mathrm{abcd}}$ \\
\hline 新疆棘豆 Oxytropis sinkiangensis & $16.04 \pm 4.11^{\mathrm{bc}}$ & $22.33 \pm 0.77^{\mathrm{d}}$ & 狐尾黄耆 Astragalus alopecurus & $25.61 \pm 11.75^{\mathrm{ab}}$ & $34.60 \pm 0.84^{\mathrm{abc}}$ \\
\hline 多叶锦鸡儿 Caragana pleiophylla & $8.93 \pm 4.24^{\mathrm{bc}}$ & $33.76 \pm 1.19^{\mathrm{abc}}$ & 弯果胡卢巴 Trigonella arcuata & $6.65 \pm 1.44^{\mathrm{c}}$ & $24.95 \pm 1.34^{\mathrm{cd}}$ \\
\hline 野火球 Trifolium lupinaster & $14.01 \pm 7.18^{\mathrm{bc}}$ & $30.04 \pm 1.49^{\mathrm{bcd}}$ & & & \\
\hline
\end{tabular}

同列不同小写字母表示差异显著 $(p<0.05)$ 。

$S R P$, seed recovery percentage; $M R T$, mean retention time. Different lowercase letters within the same column indicate significant difference $(p<0.05)$. 

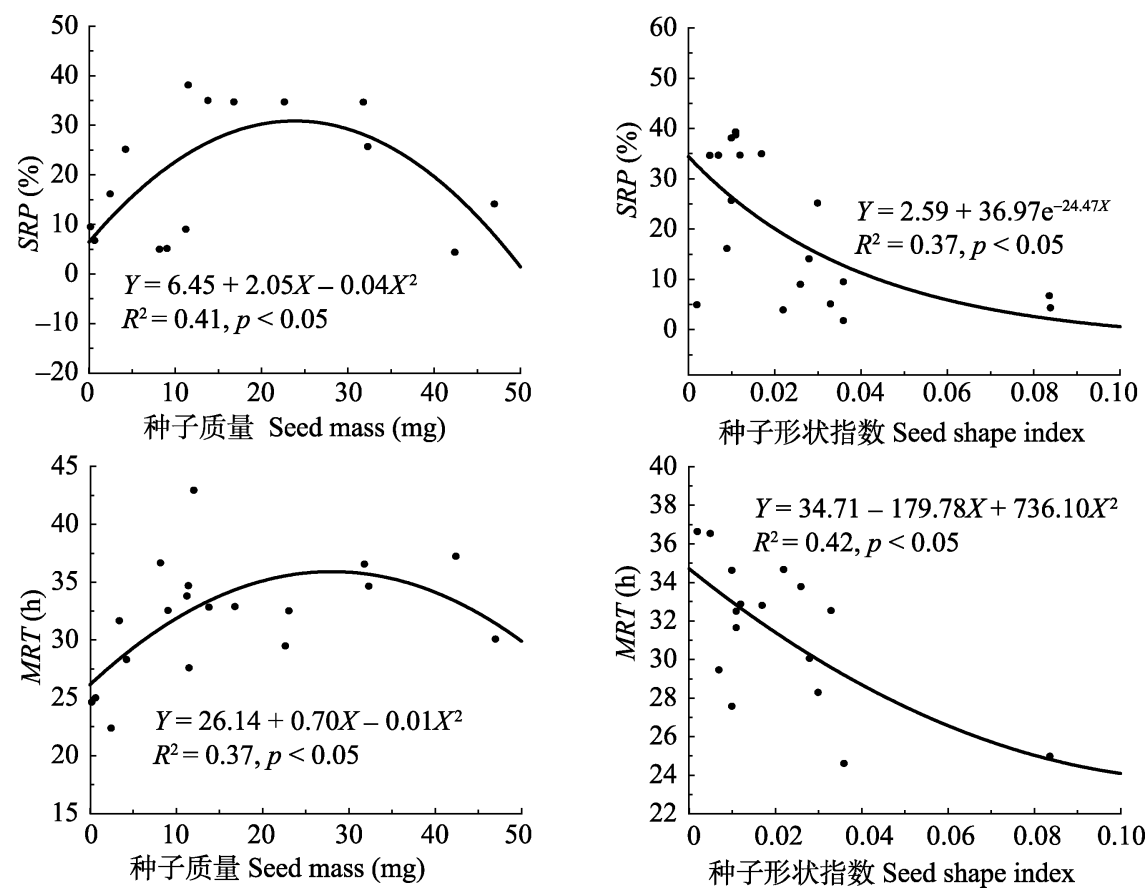

图2 种子质量和形状指数与种子回收率和平均滞留时间的关系。

Fig. 2 The relationship between seed mass, seed shape index and seed recovery percentage and mean retention time.

表3 绵羊消化前后15种豆科种子的萌发率(平均值土标准误差)

Table 3 Germination percentages of seeds of 15 leguminous species before and after sheep ingestion (mean $\pm S E$ )

\begin{tabular}{lccc} 
种名 Species & \multicolumn{2}{c}{ 萌发率 Germination rates (\%) } & \multicolumn{2}{c}{$\begin{array}{c}\text { 萌发率增值 } \\
\text { Appreciation of germination rates } \\
(\%)\end{array}$} \\
\cline { 2 - 3 } 洋甘草 Glycyrrhiza glabra & $\begin{array}{c}\text { 对照 Control } \\
\text { 消化后 After sheep ingested }\end{array}$ & 53.05 \\
草木犀 Melilotus officinalis & $15.36 \pm 5.55^{\mathrm{b}}$ & $68.41 \pm 13.86^{\mathrm{a}}$ & 17.01 \\
苦马豆 Sphaerophysa salsula & $6.67 \pm 1.16^{\mathrm{b}}$ & $23.68 \pm 1.67^{\mathrm{a}}$ & 56.01 \\
蒙古黄耆 Astragalus mongholicus & $15.00 \pm 7.00^{\mathrm{b}}$ & $71.01 \pm 3.62^{\mathrm{a}}$ & 10.67 \\
木黄耆 Astragalus arbuscula & $14.33 \pm 3.22^{\mathrm{b}}$ & $25.00 \pm 3.61^{\mathrm{a}}$ & 8.70 \\
新疆棘豆 Oxytropis sinkiangensis & $45.00 \pm 2.00^{\mathrm{b}}$ & $53.70 \pm 1.57^{\mathrm{a}}$ & 2.06 \\
多叶锦鸡儿 Caragana pleiophylla & $33.33 \pm 1.54^{\mathrm{a}}$ & $35.39 \pm 3.08^{\mathrm{a}}$ & -61.05 \\
野火球 Trifolium lupinaster & $96.22 \pm 6.33^{\mathrm{a}}$ & $35.17 \pm 3.32^{\mathrm{b}}$ & -6.90 \\
骆驼刺 Alhagi sparsifolia & $28.33 \pm 3.06^{\mathrm{a}}$ & $21.43 \pm 3.11^{\mathrm{a}}$ & 33.92 \\
山羊豆 Galega officinalis & $8.33 \pm 3.51^{\mathrm{b}}$ & $42.25 \pm 2.63^{\mathrm{a}}$ & 1.12 \\
铃铛刺 Halimodendron halodendron & $13.33 \pm 1.53^{\mathrm{a}}$ & $14.45 \pm 1.27^{\mathrm{a}}$ & 10.29 \\
野苗缩 Medicago falcata & $1.67 \pm 0.58^{\mathrm{b}}$ & $11.96 \pm 2.00^{\mathrm{a}}$ & 54.54 \\
细叶野踠豆 Vicia tenuifolia & $7.82 \pm 1.05^{\mathrm{b}}$ & $62.36 \pm 3.01^{\mathrm{a}}$ & 20.19 \\
狐尾黄耆 Astragalus alopecurus & $8.33 \pm 0.58^{\mathrm{b}}$ & $28.52 \pm 6.73^{\mathrm{a}}$ & -0.47 \\
弯果胡卢巴 Trigonella arcuata & $6.30 \pm 2.14^{\mathrm{a}}$ & $5.83 \pm 2.56^{\mathrm{a}}$ & 15.12 \\
\hline
\end{tabular}

负值表示萌发率降低。同行不同小写字母表示差异显著 $(p<0.05)$ 。

Negative value indicates that the germination rates decreased. Different lowercase letters within the same row indicate significant difference $(p<0.05)$.

(71.01\%)，最低的是狐尾黄耆 $(5.83 \%)$ 。经绵羊消化 道作用后，多叶锦鸡儿种子的萌发率由消化前的 $96.22 \%$ 显著降低至 $35.17 \%(p<0.05)$, 野火球和狐 尾黄耆的种子萌发率与消化前相比差异不显著 $(p>0.05)$; 萌发率增高的有 12 种, 占物种总数的 $80 \%$ 。

\section{3 讨论}

\section{1 种子形态学特征对 $S R P$ 和 $M R T$ 的影响}

种子是种子植物进行有性繁殖最重要的一个器 官, 也是联系上下代植物体的纽带。种子的生物学 功能主要体现在三个方面: 繁殖、散布和帮助种群

www.plant-ecology.com 
以种子形式度过不良外界环境条件(马绍宾和姜汉 侨, 1999)。种子大小和形状是对种子形态学特征最 直观的反应, 研究种子的大小和形状对于揭示种子 的传播、萌发、遗传变异和物种分类具有重要意义。 本研究通过对北疆地区15种豆科种子的大小和形状 指数进行测定, 结果表明这 15 种豆科植物种子的单 粒质量均 $>1 \mathrm{mg}$, 且 $>10 \mathrm{mg}$ 的种子有 5 种, 按照 Janzen (1977)和Jurado等(2001)的划分标准, 本研究 中的 15 种种子均为中等或偏大类型的种子; 形状指 数的测定结果表明, 除蒙古黄耆、木黄耆和弯果胡 卢巴 3 种种子外, 其余 12 种种子的形状指数均 $<0.1$, 按照Thompson等(1993)的划分标准, 这15种豆科植 物中有 $80 \%$ 的物种其种子为典型的圆(球)形种子。从 形态学特征方面来看, 本研究中的 15 种豆科种子均 为中等或较大类型的圆形种子。

植物种子经草食动物采食后的 $S R P$ 及其在动物 体内的 $M R T$ 与种子形态学特征密切相关(鲁为华等, 2013)。本研究通过将15种豆科植物种子饲喂绵羊, 并对不同时间段从绵羊粪便中回收到的种子数量的 动态变化、种子经绵羊采食后的 $S R P$ 和种子在绵羊 体内的 $M R T$ 进行统计, 结果表明, 种子排放动态符 合高斯模型: $Y=0.02+0.74 \mathrm{e}^{-0.5((X-29.61) / 9.41)^{2}}\left(R^{2}=\right.$ $0.62, p<0.0001)$, 排放高峰期集中在采食后的第 24-48 h之间，有超过 $80 \%$ 的种子在此期间排出，15 种种子的 SRP 集中在 $4 \%-39 \%$; 种子在绵羊体内的 $M R T$ 集中在22.33-37.19 $\mathrm{h}$ 时间段内。Wang等(2017) 将 13 种禾本科和 4 种豆科植物种子饲喂绵羊, 发现 种子排放规律符合高斯模型, 4种豆科种子经绵羊采 食后 $S R P$ 在 $12.6 \%-17.6 \%$ 之间, 且种子在绵羊体内 的 MRT在 27.3-42.2 $\mathrm{h}$ 时间范围内; 娜丽克斯 - 外里 等 (2016) 通过对 4 种豆科植物苦豆子 (Sophora alopecuroides)、细叶野踠豆、洋甘草和草原苜宿 (Medicago falcata) 的种子对绵羊消化道作用的反应 进行研究, 发现绵羊对这 4 种种子的排放规律均符 合高斯模型，种子的排放高峰期位于18-36 h 之间, $S R P$ 在 $4 \%-15 \%$ 之间, 种子在绵羊体内的 $M R T$ 在 29-36 h时间段内; Yu等(2012)通过将青藏高原高山 草甸 20 种植物种子饲喂藏羊, 发现种子回收率在 $0-28.1 \%$ 之间，而种子在藏羊体内的 $M R T$ 在 $12-84 \mathrm{~h}$ 之间; Manzano等(2005)研究发现, 地中海地区的灌 木种子被绵羊采食后, 种子排放规律为一条单峰曲 线, 且多数种子在采食后的 $24-40 \mathrm{~h}$ 之间被排出,
$S R P$ 在 $16 \%-23 \%$ 之间, 本实验所观察到的结果与这 些研究结果一致, 类似结果在豆科植物 Cytisus scoparius种子的绵羊消化道传播的研究中也有发现 (Manzano et al., 2005)。

本研究发现 15 种豆科植物种子经绵羊采食后, $S R P$ 随种子大小的增加呈现先增高后降低的趋势, 即大种子或小种子的 $S R P$ 都比较低。这是因为在种 子被草食动物采食和消化的过程中, 不同大小的种 子所受到的影响机制是不一样的: 大种子主要受咀 嚼过程的影响, 小种子主要受消化过程的影响 (Cosyns et al., 2005)。大种子在动物的采食过程中可 能被嚼碎, 从而降低SRP (Milotić \& Hoffmann, 2016)。在绵羊饲喂实验中, 能直接从绵羊粪便中发 现被嚼碎的Retama sphaerocarpa (单粒质量77 mg) 的种子(Manzano et al., 2005)。小种子虽然能从咀嚼 过程中成功“逃逸” (Mouissie et al., 2005a; Merelb et al., 2008), 但是小种子拥有较大的面积质量比, 这 一特点限制了其种皮厚度, 致使消化液对它们的作 用更为强烈(Manzano et al., 2005), 因此过大或过小 的种子其 $S R P$ 都比较低。也有研究表明豆科种子被 绵羊采食后, 种子大小与 $S R P$ 呈反比(Russi et al., 1992), 本研究所观察到的现象与之并不一致, 这主 要是研究对象的不同所致(Wang et al., 2017)。有研 究表明中等大小的种子被动物采食后的回收率要比 较小或较大的种子都高(Manzano et al., 2005), 本研 究的结果与之一致。主流观点认为, 圆形种子通过 动物消化道的速度要快(Mouissie et al., 2005a; 万娟 娟等, 2013), 快速通过消化道有利于提高 $S R P$ 。因为 在严酷的消化道内停留的时间过长会给种子造成致 命的伤害(Mouissie et al., 2005b; 鲁为华等, 2013), 甚至有可能将种子彻底消化掉, 从而降低了种子的 回收率。如本研究中草木犀种子在绵羊体内的 $M R T$ 最长 $(37.19 \mathrm{~h})$, 而其 $S R P$ 最低 $(4.28 \%)$ 。本研究中的 15 种豆科植物中, 有 $80 \%$ 的种子的形状指数均 $<0.1$, 为典型的圆形种子(Thompson et al., 1993), 且它们 经过绵羊采食后的 SRP 随形状指数的增加(即种子 越不规则)逐渐减少, 这一点与主流观点一致。

本研究发现 15 种豆科植物种子在绵羊体内的 $M R T$ 随种子大小的增加呈现先增高后降低的趋势, 这是因为小种子除了能在咀嚼过程中成功“逃逸”外, 还非常容易通过草食动物的瘤胃网口(娜丽克斯 外里等, 2016), 因此通过动物消化道的速度较快 
(Soons et al., 2008)。但也有研究表明种子在消化道 内的排出速率在种子大小规格上存在一个临界值, 低于这一临界值的种子经过家畜消化道的速度可能 很快, 但通过速率并非完全随着种子质量的缩小而 单调增加, 而是基本维持在稳定的水平, 并着重指 出低于这一临界值的小种子很容易通过瘤网口; 反 之, 如果种子大小高于这一临界值, 则速度可能会 变慢(D'Hondt \& Hoffmann, 2011)。但本研究也发现 大种子在绵羊体内的MRT较短, 具体原因还有待进 一步研究确定。

消化道传播是植物种子实现远距离传播的一种 重要方式(Janzen, 1984; Myers et al., 2004)。植物种 子若要成功地通过动物的消化道实现远距离传播, 则必须满足两个条件: 一是有足够数量且具活力的 种子成功地随动物粪便排出体外; 二是植物种子在 动物的消化道内要有足够长的滞留时间, 以便伴随 畜体移动而产生大尺度位移(Thomson et al., 2011)。 本研究中有 5 种豆科植物种子经绵羊采食后的 $S R P$ 超过 $30 \%$, 且种子在绵羊体内的 $M R T$ 主要集中在 22.33-37.19 h之间, 如此漫长的滞留时间为种子伴 随畜体移动实现远距离传播提供了充足的时间保 证。有研究表明, 在北美草原, 白尾鹿(White-tailed deer)每天活动范围达数公顷, 延龄草属(Trillium)种 子在其体内 $M R T$ 为9-24 $\mathrm{h}$, 种子传播距离能超过 $3 \mathrm{~km}$ (Myers et al., 2004)。当然, 种子传播距离与放 牧绵羊的管理方式有关, 在西班牙放牧体系中, 放 牧绵羊每天的移动距离为 25-30 km (Klein, 1981), 而在北疆传统放牧系统中, 放牧绵羊在草地上自由 游走, 有研究表明北疆放牧羊群每天的移动距离达 7-10 km (汪传建等, 2016), 这为豆科植物种子通过 放牧绵羊消化道实现长距离传播提供了一条潜在的 途径。

\section{2 动物消化道作用对种子萌发特性的影响}

种子经动物消化道作用后能够萌发是实现消化 道传播的前提。有研究表明, 具有休眠特性的种子 经动物消化道作用后, 其休眠特性均被不同程度地 打破(Grande \& Martinvicente, 2013)。一方面是因为 动物的啃咬和咀嚼过程可导致种子的种皮被破坏, 引起种子透水(气)性增加(Baskin \& Baskin, 1999), 另一方面, 种子在动物消化道内瘤胃消化液、微生 物(潘龙等, 2015)和众多酶类(Yu et al., 2012)的综合 作用下, 种皮软化, 透气透水性增强, 从而破除了
种子的休眠。本研究通过对 15 种豆科植物种子经绵 羊消化道作用前后的萌发率进行观测, 结果表明, 种子经绵羊消化道作用后, 有 $80 \%$ 的种子其萌发率 较对照组显著提高 $(p<0.05)$, 说明绵羊消化作用能 显著打破豆科种子物理性休眠, 这一结果与上述研 究结果一致。但本研究也发现多叶锦鸡儿对照组种 子的萌发率为 $96.22 \%$, 表明其种子的休眠率较低, 经绵羊消化道作用后, 萌发率显著降低至 $35.13 \%(p<$ 0.05), 这说明动物的消化作用并不是一味地提高种 子萌发率, 对于一些休眠率较低的种子, 消化作用 甚至能使种子失活(Gökbulak, 2002)。

草食动物对种子的消化道传播包括一系列过程, 如咀嚼和吞咽, 之后种子进入消化道沉积下来开始 真正的消化。如果是反刍类动物(如绵羊), 还要经过 反刍等二次咀嚼和吞咽, 上述过程结束以后, 才将 种子连同粪便一块排出体外。而天然草地中的豆科 植物多为草本植物(陈本建, 2008), 其种子在物理性 状上有自身的特点(数量多, 种子小, 种皮薄), 由于 草食动物取食对象的多样性和消化过程的独特性 (如反刍动物), 使得动物对此类种子的传播机制更 为复杂(Wang \& Smith, 2002)。植物种子被草食动物 采食后在消化道内的命运只是消化道传播的第一步, 真正意义上的传播还包括种子散布到外界环境后萌 发形成幼苗和植株建成等过程。而且, 在种子随粪 便排放到外界以后, 种子萌发到植株建成也具有自 身特点, 种子在粪便中的沉积可能会对萌发和植株 形态建成有利(Plumptre, 2003), 但同时也有可能存 在负面影响(牟晓明等, 2013)。因此, 种子被草食动 物摄取后是否能够实现真正意义上的传播应该包括 从种子摄入到植株成功建植的全过程, 同样, 对种 子能否成功传播的影响因素也伴随在上述整个过程 中。在这些因素当中, 种子排放地的生境对消 化道传播成功与否起决定性作用, 因为种子排放地 的生境条件能影响植物种子消化道传播的有效性 (Jordano et al., 2001; Spiegel \& Nathan, 2007; CalviñoCancela \& Martín-Herrero, 2009)。本研究中15种豆科 植物种子的采集地地势相对平坦, 整个草场植被覆 盖均匀, 群落结构复杂, 生产力水平较高, 是北疆 地区保存相对完好的放牧区域, 且在北疆传统放牧 管理方式中, 各种家畜主要处于散养状态, 放牧绵 羊在草地中自由行走采食, 人为干预较少, 种子被 排放至适宜生境的机率较大。北疆绵羊在放牧过程 
中具有择食性(间学慧等, 2009), 且绵羊消化道作用 能显著地提高豆科种子的萌发率，这为北疆地区豆 科植物种子通过放牧绵羊进行消化道传播提供了一 条有效途径。

致谢 石河子大学动物科技学院杨洁晶同学在种子 收集、绵羊饲喂和收粪试验过程中给予帮助和支持, 特此致谢!

\section{参考文献}

Baskin CC, Baskin JM (2014). Seeds: Ecology, Biogeography, and Evolution of Dormancy and Germination. 2nd edn. Academic Press, New York.

Blackshaw RE, Rode LM (1991). Effect of ensiling and rumen digestion by cattle on weed seed viability. Weed Science, 39, 104-108.

Brochet AL, Guillemain M, Gauthier-Clerc M, Fritz H, Green AJ (2010). Endozoochory of Mediterranean aquatic plant seeds by teal after a period of desiccation: Determinants of seed survival and influence of retention time on germinability and viability. Aquatic Botany, 93, 99-106.

Calviño-Cancela M, Martín-Herrero J (2009). Effectiveness of a varied assemblage of seed dispersers of a fleshy-fruited plant. Ecology, 90, 3503-3515.

Chen BJ (2008). Legume forage resources and floristic characteristics in Gansu Province. Pratacultural Science, 25(4), 42-45. [陈本建 (2008). 甘肃省豆科牧草资源及植物区 系分析. 草业科学, 25(4), 42-45.]

Cosyns E, Delporte A, Lens L, Hoffmann M (2005). Germination success of temperate grassland species after passage through ungulate and rabbit guts. Journal of Ecology, 93, 353-361.

Couvreur M, Cosyns E, Hermy M, Hoffmann M (2005). Complementarity of epi- and endozoochory of plant seeds by free ranging donkeys. Ecography, 28, 37-48.

D'Hondt B, Hoffmann M (2011). A reassessment of the role of simple seed traits in mortality following herbivore ingestion. Plant Biology, 13, 118-124.

Gökbulak F (2002). Effect of American bison (Bison bison L.) on the recovery and germinability of seeds of range forage species. Grass \& Forage Science, 57, 395-400.

Gökbulak F (2006). Recovery and germination of grass seeds ingested by cattle. The Journal of Biological Sciences, 6 , 23-27.

Grande D, Martinvicente A (2013). Endozoochorus seed dispersal by goats: Recovery, germinability and emergence of five Mediterranean shrub species. Spanish Journal of Agricultural Research, 11, 347-355.

Han JG (2007). Grassland Science. 3rd edn. China Agriculture Press, Beijing. [韩建国 (2007). 草地学. 第三版. 中国 农业出版社, 北京.]

Heidrun W, Oliver T (2008). A mechanistic simulation model of seed dispersal by animals. Journal of Ecology, 96, 1011-1022.

Janzen DH (1977). Variation in seed size within a crop of a costa rican Mucuna andreana (Leguminosae). American Journal of Botany, 64, 347-349.

Janzen DH (1984). Dispersal of small seeds by big herbivores: Foliage is the fruit. The American Naturalist, 123, 338-353.

Jaroszewicz B (2013). Endozoochory by European bison influences the build-up of the soil seed bank in subcontinental coniferous forest. European Journal of Forest Research, 132, 445-452.

Jurado E, Estrada E, Moles A (2001). Characterizing plant attributes with particular emphasis on seeds in Tamaulipan thornscrub in semi-arid Mexico. Journal of Arid Environments, 48, 309-321.

Klein J (1981). La Mesta. 2nd edn. Alianza Editorial, Madrid, Spain.

Lu WH, Wan JJ, Yang JJ, Ren AT, Yu L (2013). Review of endozoochory of plant seeds by herbivores. Acta Prataculturae Sinica, 22, 306-313. [鲁为华, 万娟娟, 杨洁 晶, 任爱天, 于磊 (2013). 草食动物对植物种子的消化 道传播研究进展. 草业学报, 22, 306-313.]

Ma SB, Jiang HQ (1999). Study on the seed weight and seed seize variation pattern and their biological significance in Podophylloideae (Berberjdaceae). Acta Botanica BorealiOccidentalia Sinica, 19, 715-724. [马绍宾, 姜汉侨 (1999). 小檗科鬼臼亚科种子大小变异式样及其生物学 意义. 西北植物学报, 19, 715-724.]

Mancilla-Leytón JM, Fernández-Alés R, Vicente AM (2012). Plant-ungulate interaction: Goat gut passage effect on survival and germination of Mediterranean shrub seeds. Journal of Vegetation Science, 22, 1031-1037.

Manzano P, Malo JE, Peco B (2005). Sheep gut passage and survival of Mediterranean shrub seeds. Seed Science Research, 15, 21-28.

Milotić T, Hoffmann M (2016). How does gut passage impact endozoochorous seed dispersal success? Evidence from a gut environment simulation experiment. Basic and Applied Ecology, 17, 165-176.

Mou XM, Yu YW, Zhang HM, Sun H, Wang HC, Xu CL, Hua LM (2013). Effects of yakdung deposition on community characteristics and niche parameters in alpine meadow. Pratacultural Science, 30，1594-1601. [牟晓明，于应文， 张红梅, 孙红, 王虎成, 徐长林, 花力民 (2013). 牦牛 粪对高寒草甸植被群落特征和生态位参数的影响. 草 业科学, 30, 1594-1601.]

Mouissie AM, Veen CEJVD, Veen GF, Diggelen RV (2005a). Ecological correlates of seed survival after ingestion by fallow deer. Functional Ecology, 19, 284-290.

Mouissie AM, Vos P, Verhagen HMC, Bakker JP (2005b). Endozoochory by free-ranging, large herbivores: Ecological correlates and perspectives for restoration. Basic and Applied Ecology, 6, 547-558. 
Myers JA, Vellend M, Gardescu S, Marks PL (2004). Seed dispersal by white-tailed deer: Implications for longdistance dispersal, invasion, and migration of plants in eastern North America. Oecologia, 139, 35-44.

Oudtshoorn KVRV, Rooyen MWV (1998). Dispersal Biology of Desert Plants. Springer, Berlin.

Pan L, Niu JL, Bu PD, Du H, Cheng JB, Sun XZ, Wang XM, Qin JJ, Yuan YM, Zhang XK (2015). Effects of saikosaponin on in vitro fermentation parameters and bacterial quantity. Acta Prataculturae Sinica, 24(6), 85-91. [潘 龙, 牛俊丽, 卜登攀, 杜洪, 程建波, 孙先枝, 王秀敏, 秦俊杰，袁耀明，张幸开 (2015). 柴胡㿝苷对体外发酵 参数及细菌数量变化的影响. 草业学报, 24(6), 85-91.]

Plumptre AJ (2003). Seed germination and early seedling establishment of some elephant-dispersed species in Banyangmbo wildlife sanctuary, South-western Cameroon. Journal of Tropical Ecology, 19, 229-237.

Ramos ME, Robles AB, Castro J (2006). Efficiency of endozoochorous seed dispersal in six dry-fruited species (Cistaceae): From seed ingestion to early seedling establishment. Plant Ecology, 185, 97-106.

Russi L, Cocks PS, Roberts EH (1992). The fate of Legume seeds eaten by sheep from a Mediterranean grassland. Journal of Applied Ecology, 29, 772-778.

Soons MB, van der Vlugt C, Van Lith B, Heil GW, Klaassen M (2008). Small seed size increases the potential for dispersal of wetland plants by ducks. Journal of Ecology, 96, 619-627.

Spiegel O, Nathan R (2007). Incorporating dispersal distance into the disperser effectiveness framework: Frugivorous birds provide complementary dispersal to plants in a patchy environment. Ecology Letters, 10, 718-728.

Thompson K, Band SR, Hodgson JG (1993). Seed size and shape predict persistance in soil. Functional Ecology, 7, 236-241.

Thomson FJ, Moles AT, Auld TD, Kingsford RT (2011). Seed dispersal distance is more strongly correlated with plant height than with seed mass. Journal of Ecology, 99, 1299-1307.

Wali N, Wang SL, Zhao G, Yang JJ, Lü YP, Lu WH (2016). Effect of sheep digestion on morphology, recovery and germinability of four Leguminous plants seed. Pratacultural Science, 33, 1566-1573. [娜丽克斯 - 外里, 王树林, 赵刚, 杨洁晶, 吕艳萍, 鲁为华 (2016). 4种豆科牧草种 子对绵羊消化道作用的反应. 草业科学，33，15661573.]

Wan JJ, Yang JJ, Ren AT, Ma CH, Yu L, Lu WH (2013). Effects of herbivores on seed germination by meta analysis. Pratacultural Science, 30，1852-1861. [万娟娟, 杨洁晶, 任爱天, 马春晖, 于否, 鲁为华 (2013). 草食动物影响 植物种子萌发的整合分析. 草业科学, 30, 1852-1861.]

Wang BC, Smith TB (2002). Closing the seed dispersal loop. Trends in Ecology and Evolution, 17, 379-386.

www.plant-ecology.com
Wang CJ, Wang WQ, Lu WH, Wen CL, Yin XJ, Zhao QZ (2016). Feed intake distribution model for herd based on grazing spatio-temporal trajectory data. Transactions of the Chinese Society of Agricultural Engineering, 32(13), 125-130. [汪传建, 王伟强, 鲁为华, 文春雷, 尹小君, 赵庆展 (2016). 基于放牧时空轨迹数据的牧群采食量 分布模型. 农业工程学报, 32(13), 125-130.]

Wang SL, Lu WH, Wali N, Ma CH, Zhang QB, Wang CJ (2017). Recovery and germination of seeds after passage through the gut of Kazakh sheep on the north slope of the Tianshan Mountains. Seed Science Research, 27, 43-49.

Wang SL, Wali N, Lu WH, Wang WQ, Wang CJ (2017). Morphological characteristics of six kinds of mucilaginous seeds and their response to digestion in rumen of sheep. Acta Prataculturae Sinica, 26(4), 89-98. [王树林, 娜丽克 斯 - 外里, 鲁为华, 王伟强, 汪传建 (2017). 六种粘液种 子性状多样性及其对绵羊瘤胃消化的反应. 草业学报, 26(4), 89-98.]

Willms WD, Acharya SN, Rode LM (1995). Feasibility of using cattle to disperse cicer milkvetch (Astragalus cicer L.) seed in pastures. Canadian Veterinary Journal, 75, 173-175.

Yan XH, An SZ, Yu X, Jia SB, Pan XD, Cheng FX, Li H (2009). Observation on grazing behavior of Kazak sheep in summer. Journal of Xinjiang Agricultural University, 32(4), 38-40. [闵学慧, 安沙舟, 余雄, 贾帅兵, 潘晓东, 程发祥, 李海 (2009). 哈萨克羊夏季牧食行为观测. 新 疆农业大学学报, 32(4), 38-40.]

Yang JJ, Wan JJ, Wali N, Ren AT, Lu WH (2015). Seed morphology and effect of sheep rumen digestion on germinability of 28 grass of Tianshan. Acta Prataculturae Sinica, 24(2)，104-115. [杨洁晶, 万娟娟, 娜丽克斯 - 外里, 任 爱天, 鲁为华 (2015). 28种植物种子形态学性状及其萌 发对绵羊瘤胃消化的反应. 草业学报, 24(2), 104-115.]

Yu L, Lu WH, Yan P, Luo LJ, Zhang QB (2014). Resources and assessment of natural grassland in Shaertao Mountains, Zhaosu County in Xinjiang. Chinese Journal of Grassland, 36(6), 4-11. [于磊, 鲁为华, 阎平, 罗良俊, 张前兵 (2014). 新疆昭苏县境沙尔套山天然草地植物 资源与评价. 中国草地学报, 36(6), 4-11.]

Yu XJ, Xu CL, Wang F, Shang ZH, Long RJ (2012). Recovery and germinability of seeds ingested by yaks and Tibetan sheep could have important effects on the population dynamics of alpine meadow plants on the Qinghai-Tibetan Plateau. Rangeland Journal, 34, 249-255.

Zhao LL, Wang ZL, Du JC, Li QF, Song CL (2007). The effect of sulfuric acid treatment on hard seeds of Melimotoides ruthenica strains. Chinese Journal of Grassland, 29(3), 73-77. [赵丽丽, 王照兰, 杜建材, 李青丰, 宋彩玲 (2007). 硫酸处理打破扁宿豆不同品系种子硬实的效果 研究. 中国草地学报, 29(3), 73-77.]

责任编委: 黄振英 责任编辑: 李 敏 